\title{
Modeling Worst-case Earthquake Accelerograms for Buildings and Structures
}

\author{
Oleg Vartanovich MKRTYCHEV \\ Research laboratory "Safety and Seismic Resistance of \\ Structures" \\ Moscow State University of Civil Engineering (National \\ Research University) \\ Moscow, Russia
}

\author{
Andrey Aleksandrovich RESHETOV \\ Research laboratory "Safety and Seismic Resistance of \\ Structures" \\ Moscow State University of Civil Engineering (National \\ Research University) \\ Moscow, Russia \\ e-mail: andrew331@bk.ru
}

\begin{abstract}
Input data necessary for synthesizing accelerograms are usually derived from seismic behavior of the construction site or from a statistical analysis of a set of recorded accelerograms which correspond to this particular construction site. As seismic performance of the site is independent of the structure which is supposed to be built on it, dynamic properties of the structure are usually not taken into account when compiling the input data. However, the available input data must necessarily contain the uncertainties generated by randomness inherent in the nature of seismic action. Hence the input data must be governed by certain laws of distribution. Therefore, we can use the input data which are possible for the construction site with a predefined probability and pose the gravest threat to the structure. In turn, in order to identify which of the accelerograms can possibly be the most dangerous for the structure, dynamic properties of the structure must be considered. This article defines a methodology for generating the worst-case accelerograms for buildings and other structures and presents a concrete example of generating a linear cantilever type system with a finite number of degrees of freedom.
\end{abstract}

Keywords-accelerogram; probability; Dirac delta function; variance; impulse response; Duhamel's integral; correlation function; seismic action; facility; target function; frequency response; energy

\section{INTRODUCTION}

The proposed methodology presumes two preparatory stages: obtaining dynamic properties of the building or structure in question and obtaining properties of the seismic action for the construction site [1]. These stages complete, a target function is constructed which is subsequently maximized while taking the imposed chance constraints into account. Based on the resulting maximization of the target function, the input data for generating earthquake accelerograms are formulated, after which the accelerograms are finally generated.

\section{IDENTIFYING DyNAMIC PROPERTIES OF BUILDINGS AND STRUCTURES}

The model of the structure is going to be represented by a cantilever-type system with $N$ degrees of freedom (Fig. 1). Data: number of nodes (floors) $N=20$, distance between nodes (floor height) $h=5 \mathrm{~m}$, node weight $m=500 \mathrm{t}$, stiffness $E I=29 \cdot 10^{9} \mathrm{kN} \cdot \mathrm{m}^{2} \quad$ (whereas masses of all nodes are equal, stiffness is evenly distributed across the beam, starting conditions are assumed to be zero).

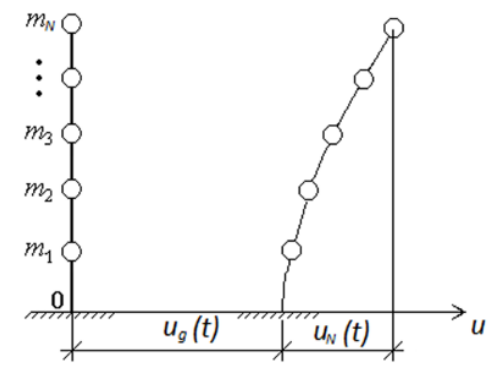

Figure 1. A system with $n$ degrees of freedom

The system's motion equation in matrix form is as follows:

$$
[m] \cdot\{\ddot{u}(t)\}+[c] \cdot\{\dot{u}(t)\}+[k] \cdot\{u(t)\}=-[m] \cdot\{l\} \cdot \ddot{u}_{g}(t) ;(1)
$$

where $[m]$ - mass matrix, $[c]$ - damping matrix, $[k]-$ stiffness matrix, $\{\ddot{u}(t)\}$ - acceleration vector, $\{\dot{u}(t)\}-$ velocity vector, $\{u(t)\}$ - displacement vector, $\{l\}$ - vector characterizing external effect, $\ddot{u}_{g}(t)$ - accelerogram.

While keeping our reasoning generic, we will involve concrete data and perform the calculation using numerical techniques offered by the Matlab suite.

The impulse and frequency response of the system representing the highest floor $\left(20^{\text {th }}\right.$ floor $)$ should be determined.

As known, the response of a linear dynamic system with a single degree of freedom (displacement in this case) can be expressed in the form of the Duhamel's integral [2]:

$$
u(t)=f(t) * h(t)=\int_{T_{M}} f(\tau) \cdot h(t-\tau) d \tau
$$

where $h(t)$ - impulse response of the system, $f(t)-$ external action, $T_{M}$ - modeling time, «*»-convolution. 
Knowledge of the impulse response of the system makes it possible to learn the system's reaction to an arbitrary effect. In turn, the impulse response is reflected by the system's reaction to the delta function (Fig. 2).

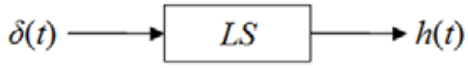

Figure 2. A linear system exposed to the delta function showcases its pulse response [3]

Formula (2) applied to a system with $N$ degrees of freedom can be rewritten in matrix form as

$$
\{u(t)\}=[h(t)] *\{f(t)\} .
$$

By applying the convolution theorem, we proceed to the frequency domain:

$$
\{U(\omega)\}=[H(\omega)] \cdot\{F(\omega)\}
$$

The expanded formula for displacement of the $i^{\text {th }}$ node, with inertial nature of the outside action taken into account, is as follows:

$$
u_{i}(t)=h_{i 1}(t) *\left(-m_{1} \ddot{u}_{g}(t)\right)+\cdots+h_{i N}(t) *\left(-m_{N} \ddot{u}_{g}(t)\right)
$$

Convolution enables us to present the formula (5) in the following way:

$$
u_{i}(t)=\left(-\sum_{j=1}^{N} m_{j} h_{i j}(t)\right) * \ddot{u}_{g}(t)
$$

Now we are introducing the notion of node-related pulse response under seismic action. $i^{\text {th }}$ - node-related pulse response under seismic action means a reaction of the system in its $i^{\text {th }}$ node exposed to soil acceleration represented by the delta function.

Let us formulate the pulse response restricted to the $i^{\text {th }}$ node via $h^{(i)}(t)$ :

$$
h^{(i)}(t)=-\sum_{j=1}^{N} m_{j} h_{i j}(t)
$$

(6) and (7) enable us to obtain the expression:

$$
u_{i}(t)=h^{(i)}(t) * \ddot{u}_{g}(t)=\int_{T_{M}} h^{(i)}(t-\tau) \ddot{u}_{g}(\tau) d \tau
$$

After transferring to the frequency domain, Formula (8) can be redesigned as

$$
U_{i}(\omega)=H^{(i)}(\omega) \cdot U_{g}(\omega)
$$

where $U_{i}(\omega)$ - Fourier transform of displacement, $H^{(i)}(\omega)$ - adjusted frequency response of the system, $U_{g}(\omega)$ - Fourier transform of the accelerogram.

Now we are going to calculate the pulse and frequency response of the system for the $i=20$ node $\left(20^{\text {th }}\right.$ floor $)$. Two cases are being considered: damping parameter $\xi_{j}=0$ (Fig. 3a) and $\xi_{j}=0,05$ (Fig. 3b). $A$ and $b$, the upper graphs of Fig. 3, present the adjusted pulse response, the next two moduli of the pulse response.
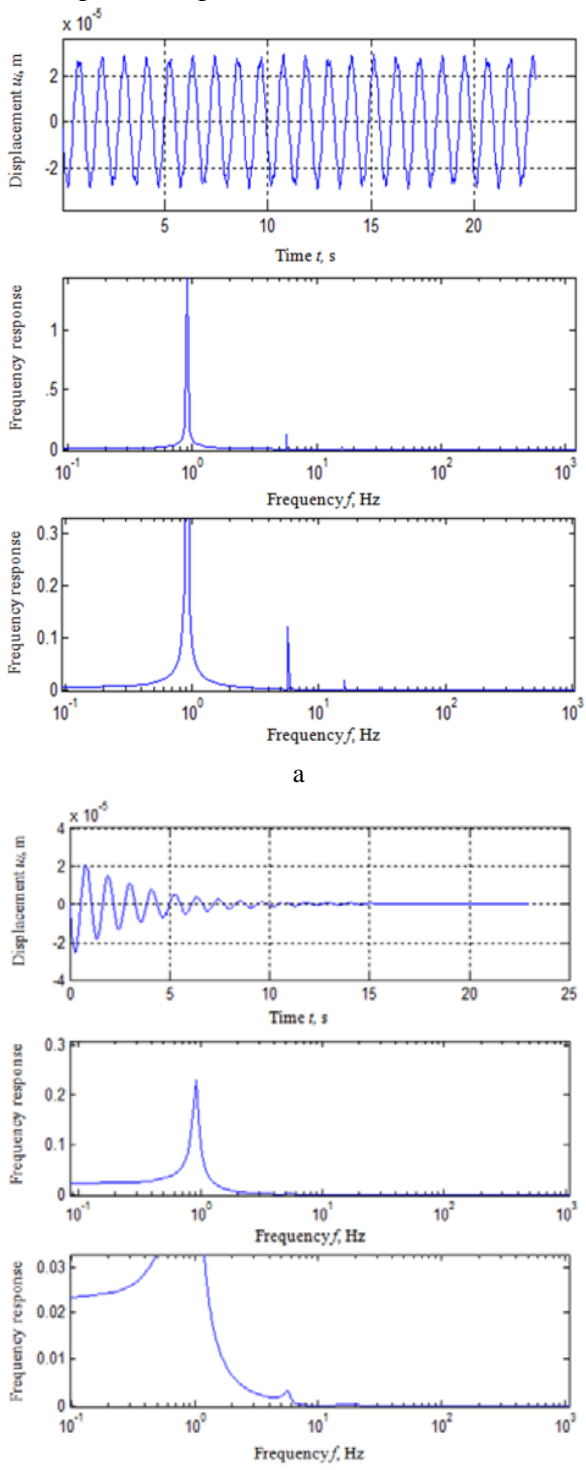

Figure 3. Adjusted pulse response and adjusted frequency response, given a) $\xi_{j}=0$, b) $\xi_{j}=0.05$ 


\section{Obtaining Properties of Seismic Action fOR A CONSTRUCTION SITE}

Properties of seismic action are obtained by statistical processing of a representative set of recorded accelerograms which correspond to a particular construction site [4].

By properties of seismic action, we mean the envelope of the accelerogram and the spectral response of the stationary part of the accelerogram (most often it is power spectrum density). Besides, the envelope characterizes both the form of variable amplitude and the exposure intensity.

The envelope and spectrum density are approximated in one way or another. In the simplest case approximation is done using analytical functions of some kind. As a result, we obtain parametrization laws which describe functions of the envelope and spectrum density, such as the position of the oscillation peak, the dominant frequency value, spectrum width etc. This paper is confined with concrete types of envelope and spectrum density functions as well as by the dominant frequency distribution law.

Let us assume that by processing a set of recorded accelerograms we have obtained the following result: the envelope is described by Formula (10) (depicted in Fig. 4a), the spectrum density is rendered by Formula (11) (see Fig. $4 \mathrm{~b})$, the law of dominant frequency distribution is Gaussian (Fig. 4c).

$$
A(t)=A_{0} \cdot \frac{t}{t_{0}} \cdot e^{-\frac{t}{t_{0}}}
$$

where $A_{0}$ - characterizes the maximum accelerations, $t_{0}-$ reflects the duration of the intensity phase. Suppose that $A_{0}=2 \cdot e^{1} \frac{\mathrm{m}}{\mathrm{s}^{2}}$ and $t_{0}=3 \mathrm{~s}$. The exposure duration is set to $23.01 s$.

$$
S(f)=\frac{2}{\pi} \frac{f_{1} f_{0}^{2}}{\left(f^{2}-f_{0}^{2}\right)^{2}+4 f_{1}^{2} f^{2}}
$$

where $f_{0}$ - the dominant frequency of a stationary random process, $f_{1}$ - the parameter reflecting the spectrum width. Let us set $f_{0}=3 \mathrm{~Hz}$ and $f_{1}=0,2 \mathrm{~Hz}$. Further on we will set other values for $f_{0}$ as well.
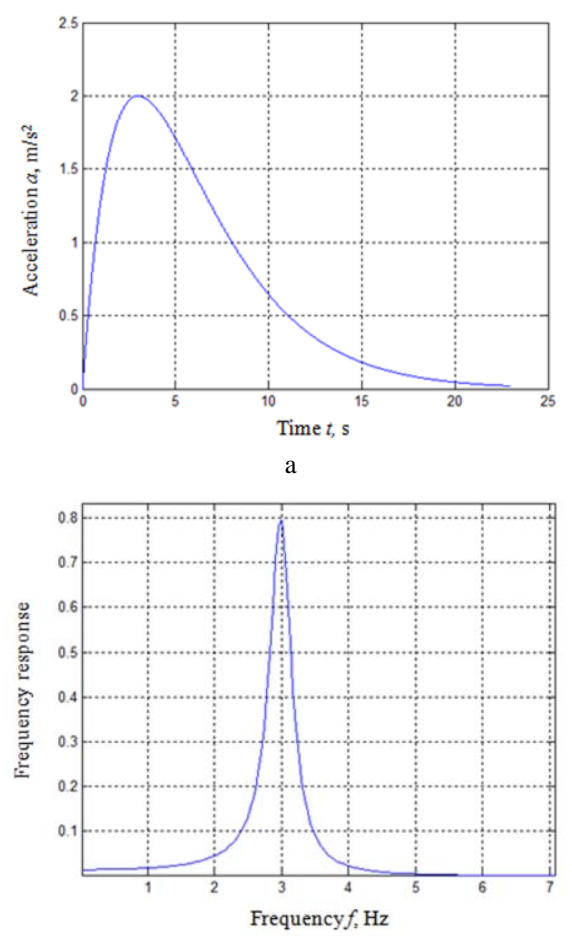

b

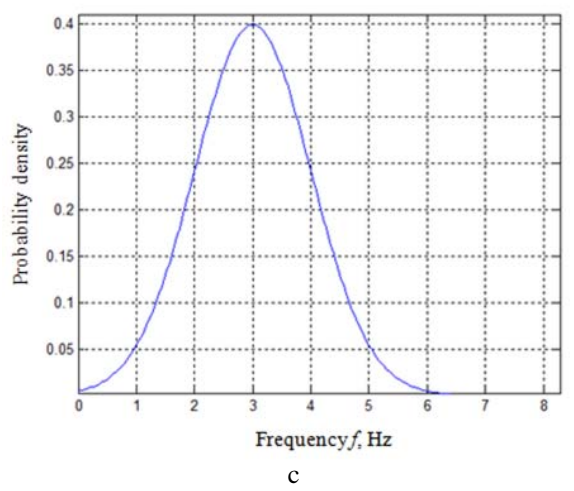

Figure 4. a) The envelope of the accelerogram, b) Spectral density of the accelerogram, c) Probability density of the dominant frequency

\section{GENERATING THE TARGET FUNCTION AND MAXIMIZING IT. GENERATING WORST-CASE ACCELEROGRAMS FOR THE SYSTEM}

Now modulus is applied to the right and left sides of the equation (9), we square it and take the integral:

$$
\int_{-\infty}^{\infty}\left|U_{i}(\omega)\right|^{2} d \omega=\int_{-\infty}^{\infty}\left|H^{(i)}(\omega)\right|^{2} \cdot\left|U_{g}(\omega)\right|^{2} d \omega
$$

By dividing the equation (12) by $2 \pi$ and applying Parseval's theorem [3], the $i$-node displacement energy is obtained (in terms of the signal theory): 


$$
E_{U_{i}}=\frac{1}{2 \pi} \int_{-\infty}^{\infty}\left|H^{(i)}(\omega)\right|^{2} \cdot\left|U_{g}(\omega)\right|^{2} d \omega .
$$

Moreover, it is known that this energy is equal to the non-normalized variance $D_{U_{i}}=E_{U_{i}}$.

Then the location parameter $\left|U_{g}(\omega, \Omega)\right|^{2}=\left|U_{g}(\omega-\Omega)\right|^{2}$ is introduced into the function.

The location parameter of the function $\left|U_{g}(\omega, \Omega)\right|^{2}$ can be interpreted as a shift of the dominant frequency around which the spectrum is formed.

After reversing the location parameter $-\Omega \rightarrow \Omega$ the variance can be formulated as a function of the $\Omega$ argument:

$$
D_{u_{i}}(\Omega)=\frac{1}{2 \pi} \int_{-\infty}^{\infty}\left|H^{(i)}(\omega)\right|^{2} \cdot\left|U_{g}(\omega+\Omega)\right|^{2} d \omega
$$

The correlation function of the two functions $\left|H^{(i)}(\omega)\right|^{2}$, $\left|U_{g}(\omega)\right|^{2}$ is given by Formula (14).

For the system's response energy to be the highest possible, we must pick the location parameter $\Omega$ so that the correlation between the functions $\left|H^{(i)}(\omega)\right|^{2}$ and $\left|U_{g}(\omega)\right|^{2}$ is the highest possible.

So, the variance (14) is the target function which must be maximized $D_{u_{i}}(\Omega) \rightarrow \max$. The target function should be maximized with constraints imposed on the location parameter $\Omega$. These constraints are as follows.

Two auxiliary frequencies $\omega_{a}, \omega_{b}$ can be introduced such that

$$
\int_{\omega_{a}}^{\omega_{b}} f\left(\omega_{d}\right) d \omega_{d}=P_{c} ; \omega_{a}<\omega_{d}<\omega_{b} ;
$$

where $\omega_{d}$ - the dominant frequency, $f\left(\omega_{d}\right)$ - probability density of the dominant frequency, $P_{c}$ - the predetermined probability value (we set $P_{c}=0.9$ ).

The location parameter $\Omega$ may take values which cause the newly obtained dominant frequency to belong to the interval $\left(\omega_{a}, \omega_{b}\right)$ :

$$
\begin{aligned}
& \left\{\begin{array}{l}
\omega_{d}+\Omega>\omega_{a} ; \\
\omega_{d}+\Omega<\omega_{b}
\end{array} ; \Rightarrow\left\{\begin{array}{l}
\Omega>\omega_{a}-\omega_{d} ; \\
\Omega<\omega_{b}-\omega_{d} ;
\end{array} ;\right.\right. \\
& \text { or } \omega_{a}-\omega_{d}<\Omega<\omega_{b}-\omega_{d}
\end{aligned}
$$

Thus, we need to shift the original spectral density of the seismic action so that, on the one hand, it causes the most intense response of the system and, on the other, this shift must fit into the criterion based on the dominant frequency distribution law which was obtained for this particular construction site.

The accelerograms which were generated based on the newly obtained spectral density will be the most unfavorable for this system. At the same time, their probability for this construction site will be no less than $1-P_{c}$.

The next step would be numerical calculations with and without damping. Accelerograms will be generated using the shaping filter method. For more detailed information on the generation methods see [4-6].

Calculations for the system with no damping $\left(\xi_{j}=0\right)$ are performed with $\omega_{d}=2 \pi \cdot 3 \mathrm{rad} / \mathrm{s}$.

Figure 5 displays the probability density of the seismic action (red curve), adjusted frequency response of the system (blue curve), original spectral density of the seismic action (black dotted line), spectral density of the seismic action obtained using the criterion (black solid line). The yaxis of spectral density and probability density was magnified for visual convenience (on the graph only).

Calculations for the system with no damping $\left(\xi_{j}=0\right)$ were done with $\omega_{d}=2 \pi \cdot 4,9 \mathrm{rad} / \mathrm{s}$.

All designations in Fig. 7-8 are similar to those of Fig. 5-6.

The analysis of the graphs for the case $\omega_{d}=2 \pi \cdot 3 \mathrm{rad} / \mathrm{s}$ allows us to make the following observations. The first natural frequency belongs to the interval $\left(\omega_{a}, \omega_{b}\right)$, and this frequency faced the shift of the original spectral density in such a manner that by multiplying the spectral density of the adjusted pulse response by the spectral density of the exposure we got the largest area under the resulting curve (which caused the first natural frequency to resonate).

By analyzing the graphs for the case $\omega_{d}=2 \pi \cdot 4,9 \mathrm{rad} / \mathrm{s}$, we can conclude that the first natural frequency does not belong to the interval $\left(\omega_{a}, \omega_{b}\right)$, while the second one does. The shift of the original spectral density headed towards the second natural frequency and caused it to resonate.

The calculations for the system with damping taken into account are presented below. All designations in Fig. 9-10 are identical to those of Fig. 5-6. 


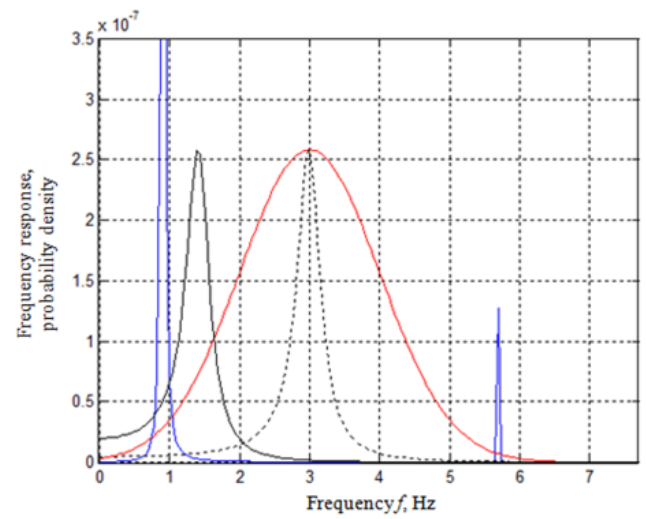

Figure 5. Shift of the original spectral density of the seismic action
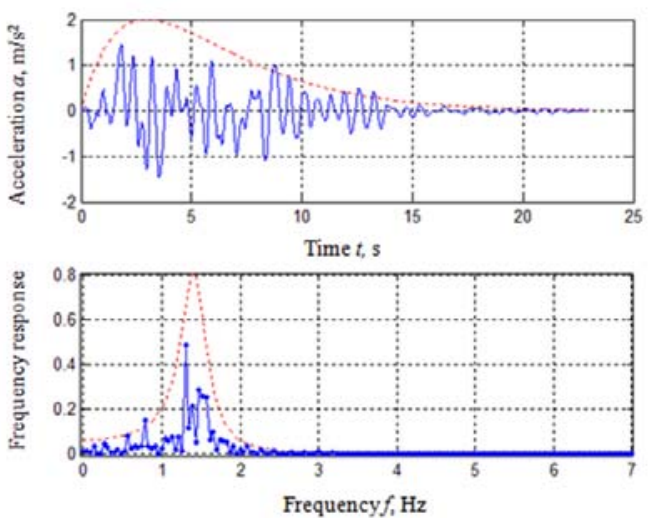

a
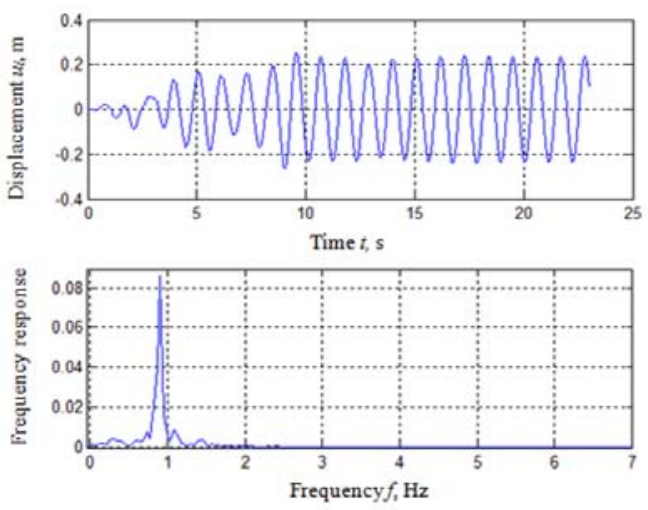

$\mathrm{b}$

Figure 6. a) The resulting worst-case accelerogram, b) Displacement of the 20th floor

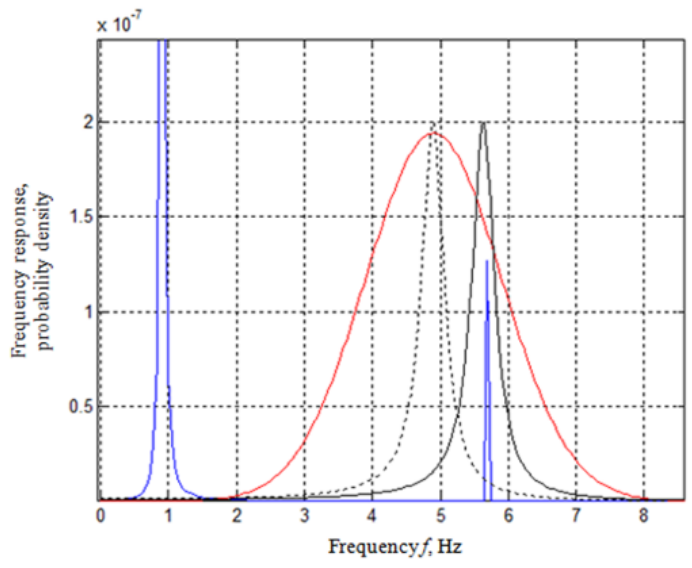

Figure 7. Shift of the original spectral density of the seismic action
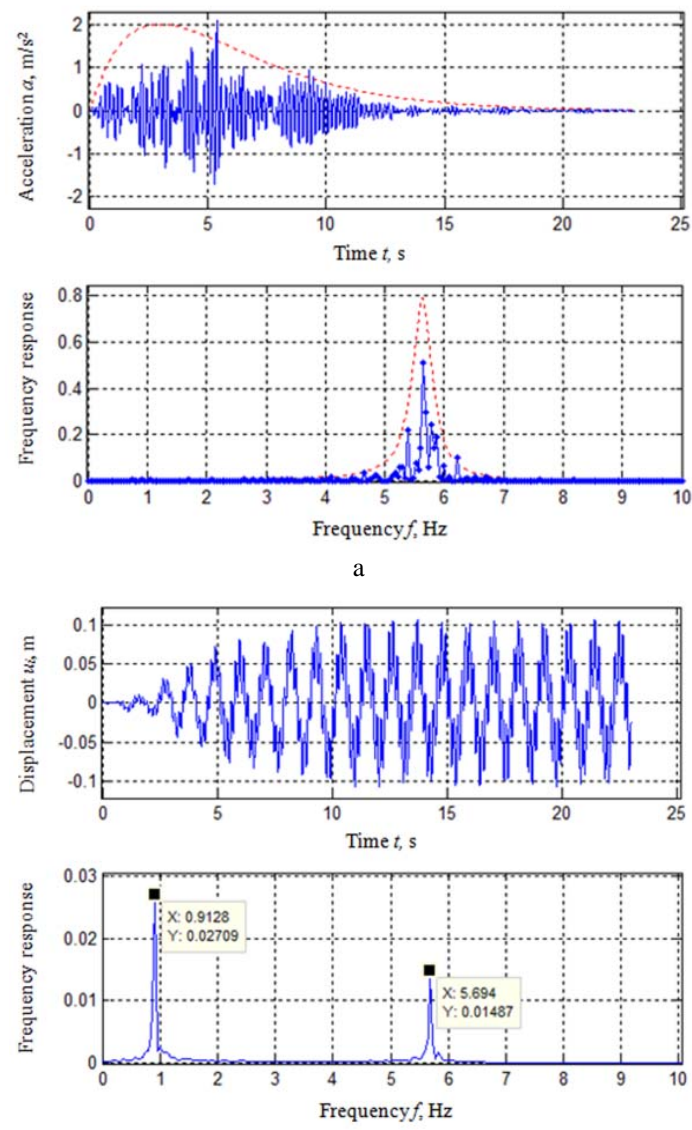

Figure 8. a) The generated worst-case accelerogram, b) Displacement of the 20th floor 


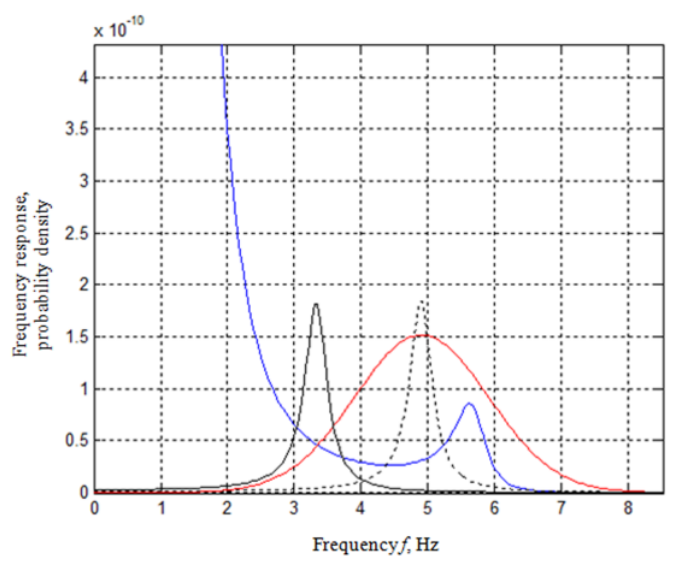

Figure 9. Shift of the original spectral density of the seismic action
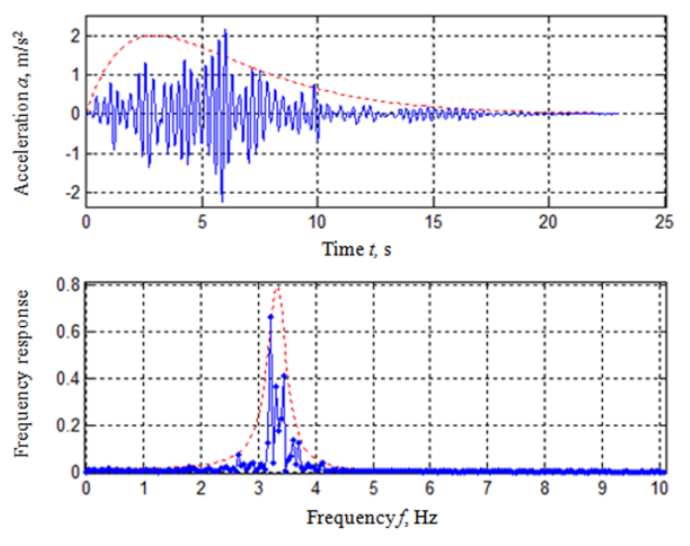

a
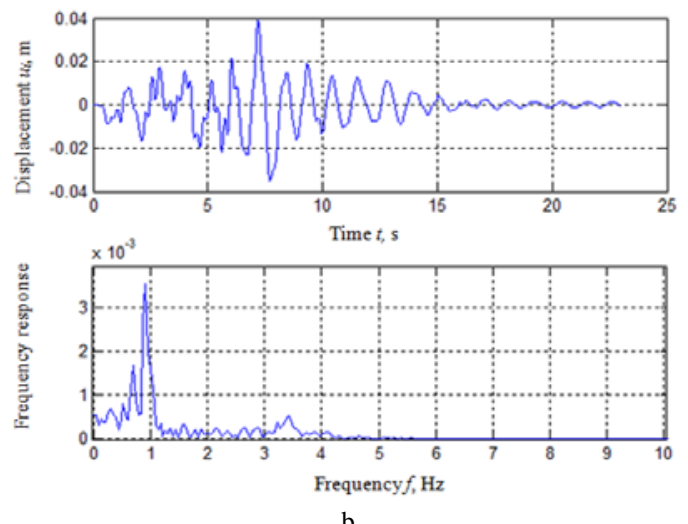

b

Figure 10. a) The generated worst-case accelerogram, b) Displacement of the 20th floor
After analyzing the graphs for the case $\omega_{d}=2 \pi \cdot 4,9$ $\mathrm{rad} / \mathrm{s}$, we can see that the original spectral density shifted towards the first natural frequency as opposed to the case of the system with no damping. This can be explained by the fact that the left branch of the resulting spectral density multiplied by the adjusted pulse response of the system contributes significantly to the energy of floor displacement. This energy is larger than the energy that would arise with the shift of the spectral density directed towards the second natural frequency of the system.

\section{CONCLUSION}

The methodology laid down in this paper can be applied in practice for designing buildings and facilities located in earthquake-endangered areas. This methodology is potentially generalizable, e.g., the target function can be viewed as a function of multiple parameters like shift of the dominant frequency, extension and contraction of spectral density, up- and downscaling of intensity etc. Besides, the constraints imposed on these variables must be determined by the statistically processed seismic data.

\section{ACKNOWLEDGMENT}

This study was performed with the support of the RF Ministry of Education and Science, grant No. 7.2122.2014/K.

\section{REFERENCES}

[1] R.Clough, J. Penzien Dynamics of structures [Dinamika sooruzheny], Stroyizdat, Moscow, 1979, 320 p.

[2] O.V. Mkrtychev, A.A. Reshetov A methodology for modeling worstcase earthquake accelerograms [Metodika modelirovaniya naiboleye neblagopriyatnykh akselerogram zemletryasyeny], Industrial and Civil Construction [Promyshlennoye I grazhdanskoye stroitelstvo], 2013, no.9, pp. 27-29.

[3] O.V. Mkrtychev, R.V. Yuryev Designing structures for seismic exposure using synthesized accelerograms [Raschet konstruktsy na seismicheskie vzainodeystviya s ispolzovaniem sintezirovannykh akselerogram], Industrial and Civil Construction [Promyshlennoye I grazhdanskoye stroitelstvo], 2010, no. 6, pp. 52-54.

[4] Cacciola Pierfrancesco A stochastic approach for generating spectrum compatible fully nonstationary earthquakes, Computers \& Structures, Volume 88, Issues 15-16, August 2010, pp. 889-901.

[5] Sanaz Rezaeian, Armen Der Kiureghian Stochastic Modeling and Simulation of Ground Motions for Performance-Based Earthquake Engineering, Pacific Earthquake Engineering Research Center College of Engineering University of California: Berkeley, 2010, 201 p.

[6] A.B. Sergiyenko Digital Signal Processing [Tsifrovaya obrabotka signalov], Piter, St Petersburg, 2002, 606 p. 